\title{
Application of the circle beauty in the environmental design of Lin-gang Special Area of Shanghai
}

\author{
Hongbo $G e^{1, *}$ \\ ${ }^{1}$ Shanghai Jian Qiao University, College of Art and Design, 201306 Shanghai, China
}

\begin{abstract}
Dishui Lake, an artificially excavated circular lake, has become a landmark landform in the Lingang Special Area of Shanghai. It contains the green ecological concept of the harmony between human and nature at the beginning of the design, and establishes a relatively perfect lighthouse coordinate for the grand blueprint of the Lin-gang Special Area. This paper takes the application of circle in environmental design as the breakthrough point, and uses the similarities of Qinglong Town site for reference, and expounds the application of the beauty of circle in environmental design to show the concept of green ecology, so as to provide an accurate connection between sea and land for the excellent cultural inheritance of the Chinese nation.
\end{abstract}

\section{Introduction}

This article is the opening work for a series of $\mathrm{R} \& \mathrm{D}$ in urban planning and design in the Lin-gang Special Area in Pudong Shanghai, it is aiming to dig out and tease out the metaphysical " Circular " philosophical ideas in traditional culture, and to use this as a driving force for $\mathrm{R} \& \mathrm{D}$, making suggestions and opening up the way for culture to lead the high quality development of the free trade zone, it also provides some necessary basic knowledge and energy for Lingang to develop into a pilot and wind vane for the rapid economic development of the Yangtze River Delta in the future. Therefore, we try to clarify its basic theory and ideological context from the level of cultural gene.

From the perspective of the local historical context and the characteristics of the landscape and environment, this article mainly talked about the relevant thoughts about the regional construction characteristics and design-art of Lingang Special Area. In particular, it summarized and discussed the deep cultural source of the standard landscape of the new coastal area: The circular beauty of Dishui Lake(Figure 1). In order to lay the theoretical foundation for the future urban construction of the new coastal area, In this way, the outstanding and profound traditional culture of the motherland will be restored and serve the current economic and cultural construction. If there is anything wrong with the following discussion, please seek expert criticism and criticism.

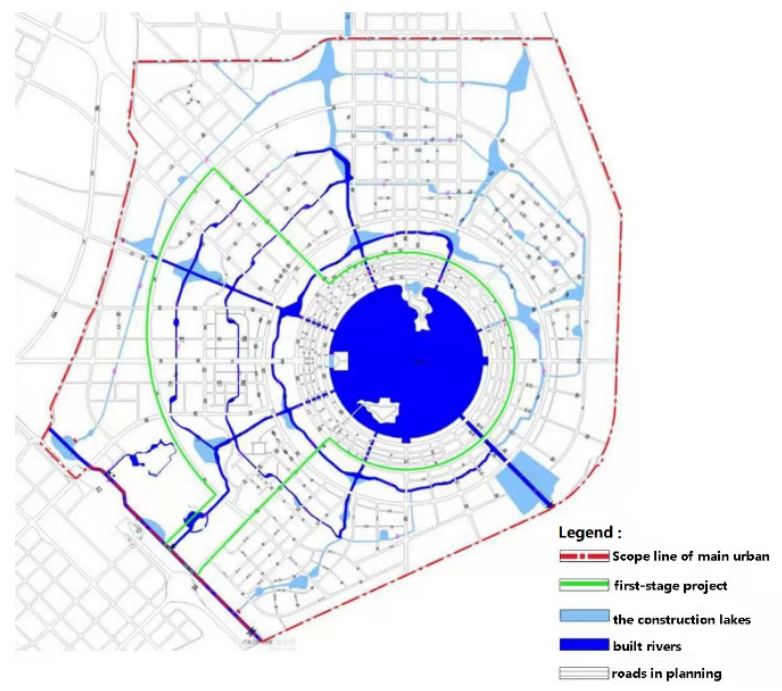

Fig.1 Associated drainage map of Dishui lake

\section{Local historical and cultural background}

On December 14, 2019, at the world archery forum held by Shanghai University, there was a research report titled "Qinglong Town_— the ruins of the port on the Sea Silk Road", which brought our thoughts back to the important foreign trade port of Shanghai and Predestined cause of towns in the Tang and Song Dynasty.

The ruins of Qinglong town in Qingpu District of Shanghai City, Baihe Town, were one of the top ten archery findings in China in 2016. In particular, they found the octagonal seven story Longping Temple Pagoda with the function of the navigation tower in the Northern Song Dynasty. The unearthed archaeological relics are consistent with the literature records. These new materials 
showed that Qinglong town was an important part in the development history of Shanghai city. ${ }^{[1]}$ The report was from the research achievement of the professor Chen, who said: according to the records, Qinglong town rose in the Tang Dynasty and was prosperous in the Song Dynasty. The literature had proven the importance of Qinglong town as the earliest foreign trade port in Shanghai city. ${ }^{[2]}$ The most important thing was that the research results confirmed that Qinglong town was one of the important port of the Sea Silk Road in the Tang and Song Dynasty. However, in the end of the Song Dynasty, due to the growing congestion in the Wusong river, the ship could no longer flow into the port of Qinglong town through the Wusong river. Its port function was gradually replaced by the later Shanghai Town, and the once prosperous Qinglong town was no longer prosperous. ${ }^{[3]}$

Qinglong town was the ruins of the port on the Silk Road on the sea, and it was the earliest foreign trade port in Shanghai. The status and function of Lin-gang Special Area was exactly similar to the previous Qinglong town. Therefore, Lingang has formed a good echo with history in terms of economic and cultural development. The view of the Dishui Lake in Lingang was just in line with the ancient people's thoughts and application of "circle". From the ancient ancestors' thought about the image of the circle, we began to think about the cultural philosophy and art aesthetic from the "circle" of the Dishui Lake in Lingang new port district, hoping to add some energy of culture art to the development of the new district(Figure 2).

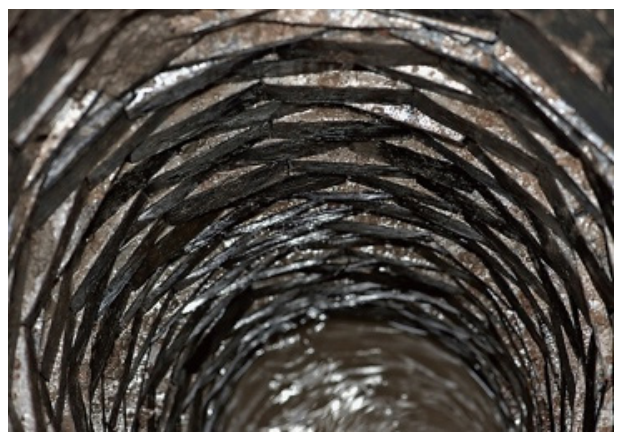

Fig.2 Wells from the Tang and Song dynasties found at the Qinglong Ruins

\section{Analysis of the circle image}

Since the symbolic landscape of the circular Dishui lake has been included in the overall planning of the New District of Lingang, we should make full use of this local image project to further dig deeper into the history and the real cultural meaning of this area, especially to study the scientific problems related to circles, such as the philosophy, cultural nature, artistic aesthetic and other relevant problems, from the perspective of culture or even gene Starting with it, the dream of the sea areas where the people were rich and the country was strong and the cultural self-confidence was plump.

It was well known that one of the main contents of the basic spirit of Chinese culture was "the harmony between human and nature", (the other three are "people oriented", "vigorous and promising" and "Respecting harmony and balance". ${ }^{[4]}$ As the leading culture in ancient China and the traditional culture spreading throughout the whole society, the thought of "the harmony between human and nature" was not only rich in content, but also complex in its own development. We should analyze the problem according to the facts. ${ }^{[5]}$ It was true that only circle could really be able to accommodate all kinds of things.

In the ancient Chinese literature, "Mencius" said, "Even if one has such good eyesight as Li Lou, is so numble-figured as Gongshuban, he can't draw a square or a circle without a pair of compasses or a crooked ruler. "What he meant was that no matter how smart and even fantastic you were, you couldn't complete the square and circle drawings without appropriate tools. While the "rules" and "rules" were the materialized crystals in the system of math and divinity.

In the west, more than 2500 years ago, when the ancient Greek sect was born in the world, the earliest Pythagorean School of Philosophy believed that the final essence of reality was numbers. Therefore, they worshiped the science and math, even their successors insisted on believing that the nature of the universe must be digital. ${ }^{[6]}$ The harmonious theory of math and beauty of the Pythagorean School also directly affected the art law of the divine temple building in the ancient Greek times. ${ }^{[7]}$ In summary, let's give a typical example to verify the fact that the Tai Chi formula exists in the ancient world.

\section{Cultural prototype and the circle in nature}

In recent years, some scholars had shown that the Tai Chi formula had once been a common phenomenon in the world. ${ }^{[8]}$ its cultural content should come from the scope of divinity, and astronomy should only be derived from this process of accompanying. That was to say, because of the low production at that time, people only prayed to God. In fact, they watched the heavenly phenomenon, calculated and drew the figures for the survival of the race. The historical relic of the heavenly vision was basically the same as that of the ordinary people who were still left behind to watch the palm, watch the constellations and predict the fortune. Due to the fact that the eastern culture had a long history with the concept of "round sky and square earth", especially the concept of "round sky", which had always dominated the Metaphysical view of the world outlook of the Huaxia ancestors, the "circle" had a deep inner connection with the prototype of the culture.

Then, where did this prototype (the circle is foundation of the Tai Chi diagram) come from?

In fact, there had been some hints before. For example, the sun, moon, stars, and the stars were the original microcosm of the stars in the universe. This was because once the objects in the space of the star rotated automatically and were affected by the airflows, the final ideal result could only be circles.It could be said that almost all the planets were like this. Even the explosion of the sun could only disperse at a certain distance.Math and geometry proved that a circle could accommodate everything as much as possible between the same area, because the center point spread in the same distance. If the sun erupted like that, it would be in accordance with the 
economic law and save time and energy.Otherwise, no matter how high its quality was, it would still lose balance and cause destruction.

In a word, from the spirit body of eastern culture to the western philosophy, from astronomy, math to laws of several laws, from the symbolic meaning of the Tai Chi diagram to the literary content of the circular pattern, all the examples were just a brief introduction.

\section{The current situation of urban construction in Lin-gang New District}

On August 25, 2009, according to the "notice of the Ministry of water resources on the announcement of the ninth batch of National Water Conservancy Scenic Spots", the Dishui Lake scenic spot in Pudong New Area officially became a national water scenic spot (Figure 3).From June 26, 2002 to October 6, 2003, the construction of the artificial lake was completed. After the Dishui Lake was completed, it became a unique ecological scenery in the new coastal area. The circular shape of the Dishui Lake was also a common form in the nature. The city planning of the Lin-gang new coastal area with the Dishui Lake as the core was to combine this shape with the city's formation. Just like water dripped into the lake, the ripples extended to the surrounding, which was in line with the traditional culture of the eastern civilization.

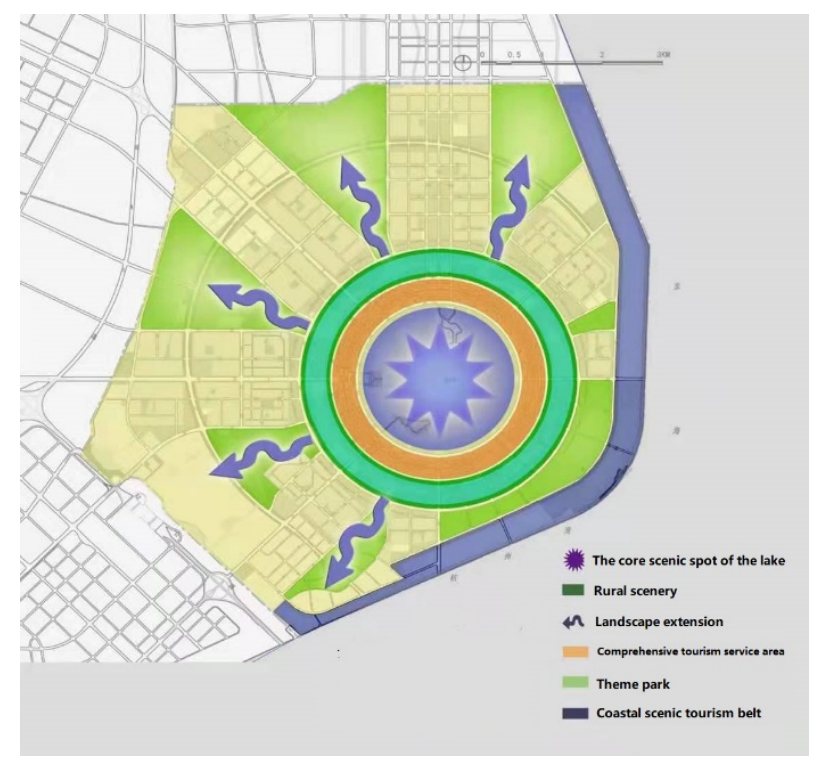

Fig.3 Planning and layout of water conservancy scenic spots of Dishui lake

The circular tension determined that its radiation was evenly divergent in all directions, which was the same as the location of the Lin-gang new coastal area. With the Lin-gang new coastal area as the center, it radiated the inland and extended to the sea. The four rivers surrounding the Dishui Lake represented the meaning of the four seasons of the year. The seven shooting river courses lifted up the shiny pearl in the Dishui Lake. The small lake formed by the junction of the river of light and the river of ripples was like a small pearl surrounding the Pearl. It fully displayed the design concept of the general planning of the new urban area of Lingang. The main city was born from a drop of water from the sky, falling into the quiet lake, and the ripples spread out in circles, forming the city's spatial layout and texture, and becoming the main guiding structure of the main city (Figure 4).In the circular culture, these radiation forces offset each other. In this case, they could even show the quiet and steady characteristics of the circle. Compared with other shapes, the circular shape was the most striking, independent and distinct.

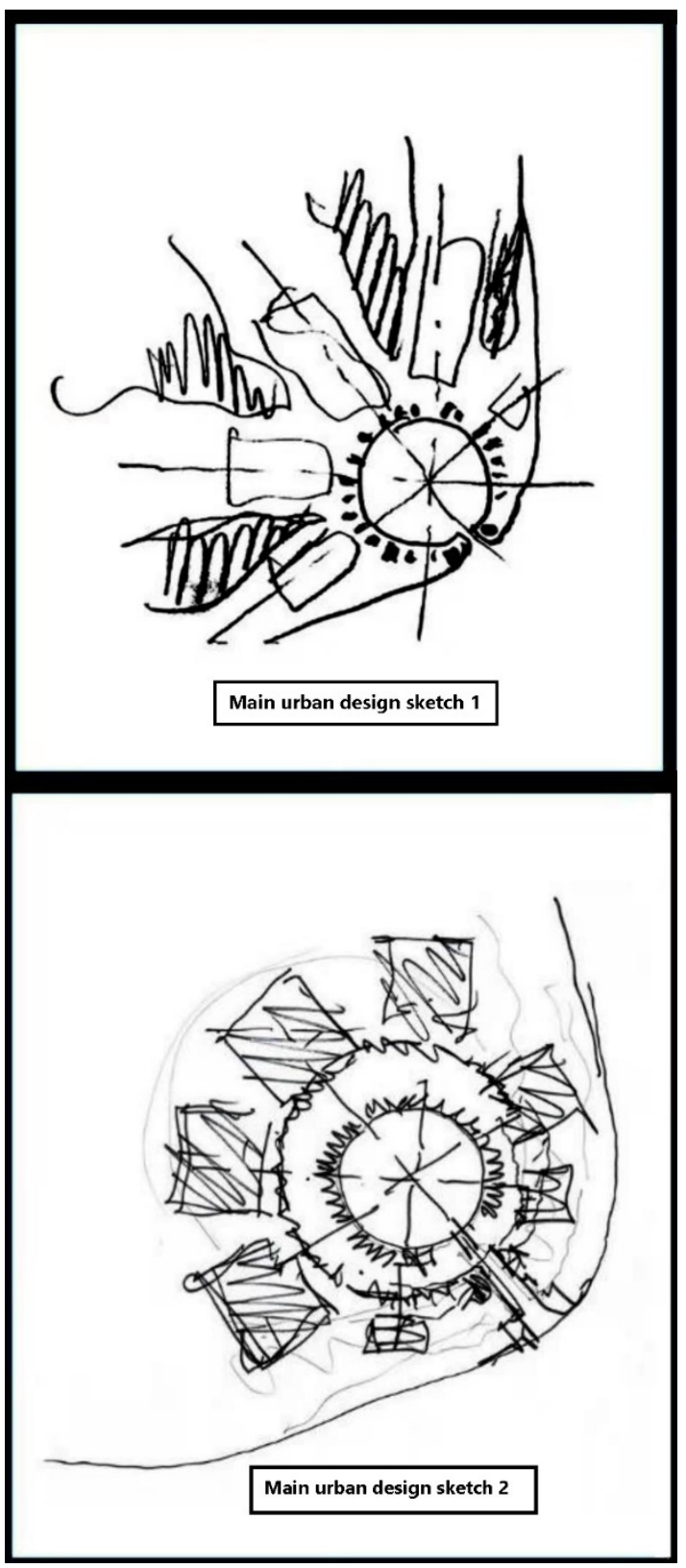




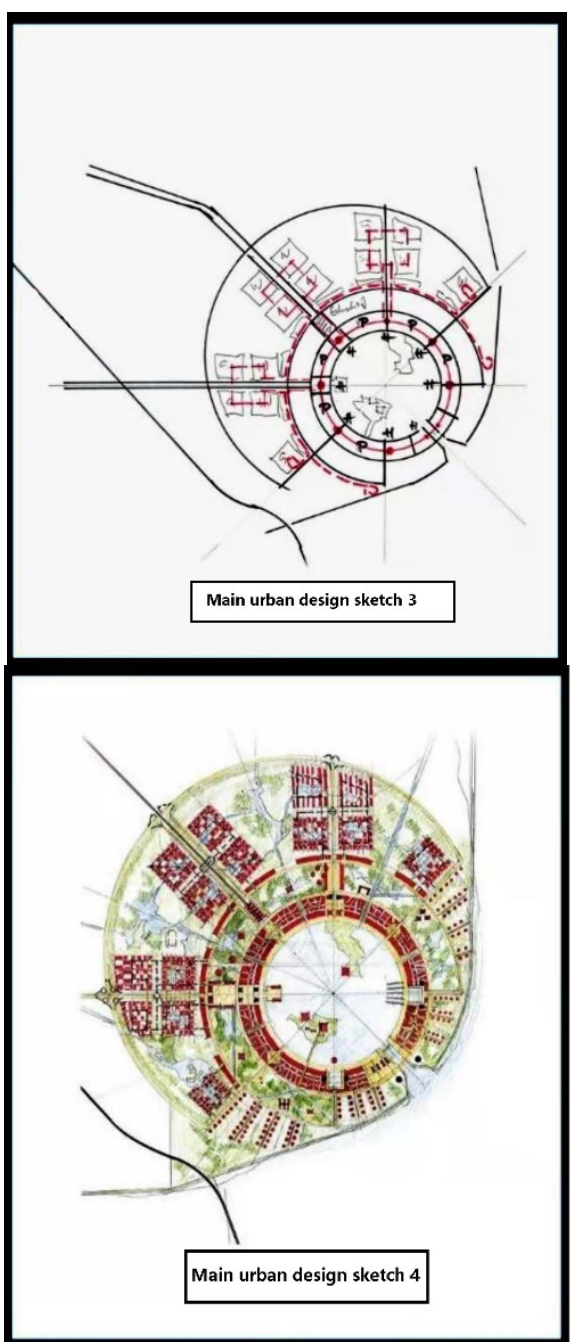

Fig.4 Main urban design hand-painted original manuscript

Because of the strong unity and integrity of the circle, its form was too independent. When it was placed together with other shapes, it was often the most eye-catching and distinct independent. Besides, the circle also had plump, complete, close, United, full and rigorous symbolic meaning.

\section{Summary}

After the preliminary discussion, the brief introduction was as follows: the circular Dishui Lake dug out by people in the sailing Lin-gang new coastal area (including the ring road around the lake) perfectly inherited the faith main body of the outstanding Chinese School of literature - "the harmony between human and nature", "round sky and square earth", which were extremely important traditional core value. We could said that the circular beautiful life lake laid the basic style and strategic direction of Lingang urban construction planning and layout. It is worthy of the good news that designers cherish the Chinese civilization and deeply understand the historical and aesthetic heritage of the oriental nation. It was not only continuing and creating an ideal atmosphere of auspicious and perfect environment in the free trade area, but also firmly maintaining the fair competition system of the modern rules, and creating a wide channel for entrepreneurs.
Moreover, the round lake not only implied the fairness and the stability of the rules, but also symbolized the perfect opening posture of the economic and cultural strategy of the free trade zone. This kind of profound design blueprint and high-level strategy could be said to be applauded. At the same time, it even encourage the successors like us, to make unremitting efforts for this, and urge all the Lingang people to work hard and to create more brilliant achievements with the broad-minded heart and indomitable spirit to bring honour to the new century and new home.

\section{References}

1. Chen Jie, "Qinglong Town -- the ruins of the port on the Silk Road on the sea", the fourth world archery Forum -- Shanghai -- the view of archery in the city and the world ---- the common future of human beings. [He gave a speech on the afternoon of December 15, 2019, and see also page 56-57 of the meeting manual].(p.56-57).

2. Chen Jie, "the history of Qinglong town and the significance of the archery discovery", Shanghai Museum, "Shanghai ancient harbor archery: the archery and discovery of Qinglong Town", Shanghai ancient book press, April 2017, (p.001-003).

3. Chen Jie, "the history of Qinglong town and the significance of the archery discovery", Shanghai Museum, "Shanghai ancient harbor archery: the archery and discovery of Qinglong Town", Shanghai ancient book press, April 2017,(p.005).

4. Zhang Dainian \& Fang Keli, "an introduction to Chinese culture", Beijing Normal University press, January 2004,(p.286).

5. Zhang Dainian \& Fang Keli, "an introduction to Chinese culture", Beijing Normal University press, January 2004,(p.289).

6. Stokes,P., "100 Great Philosophers ", translated by Chen Lili,checked by Fang Xianghong, Fudan University Press, August 2015,( p. 004-005).

7. Fred S. K et al., "Gardner' s art through the ages", Translated by Zhu Di and Zhou Qing et al, China Youth Publishing House, June 2007, (p. 126129).

8. Zhai Yuzhong, "genes of human civilization", Central Compilation and Translation Press, October 2017. 